\title{
Structures under scrutiny
}

\author{
Structural biology advances have democratized access to biomolecular snapshots, but high standards \\ must be maintained to ensure their utility.
}

Protein crystallography has been pivotal in defining the landscape of modern chemical biology. Visualizing the detailed architecture of proteins, nucleic acids and other biomolecules has shaped our molecular understanding of life and inspired countless mechanistic investigations. Given the sometimes extensive engineering required to generate crystallizable constructs, as well as an increased appreciation for the role of dynamics in biological function, scientists are generally mindful of the caveat that these structural representations reflect only one possible state of the molecule. However, not enough scientists are aware that these structures may-in the worst case-not represent any state of the molecule, as a result of errors in interpretation or deficiencies in validation of the structural data. A renewed commitment to scientific, educational and publication standards will be needed to ensure the ongoing utility of our structural data and the veracity of the research that builds on those structures.

The number of Nobel Prizes related to crystallography testifies to the technical advances that have transformed the field and the importance of the knowledge they have revealed. Yet specialists have always been wary of the damage that can be done by incorrect structures; as the Protein Data Bank (PDB; http://www.rcsb.org) grew from seven structures at its founding in 1971, discoveries of substantial errors in the database worried practitioners in the field. In one cautionary essay, Brändén and Jones (Nature 343, $687-689,1990)$ warned researchers: "It is the crystallographer's responsibility to make sure that incorrect protein structures do not reach the literature." These concerns motivated the development of a cadre of automated tools to assist in determining and validating structures. With confidence renewed, crystallographers were poised to take advantage of sequences coming from genomics initiatives, leading to both improved methods and a wealth of new structures.

These advances have enabled ever more scientists to try their hand at crystallography, as reflected in the growth of the PDB to more than 100,000 structures. However, it is critical that newcomers to the field educate themselves on proper procedures of structural refinement as well as about the sometimes subtle decisions that can lead to errors. For example, incorrect timing of ligand placement in a structural refinement can result in misleading models. Further, because many current refinement tools do not adequately handle certain post-translational modifications or small molecules, structural problems may be missed. Even when appropriate refinement and validation steps could be used, Cowtan and colleagues note, these procedures are often bypassed, with the result that many of the carbohydrate structures in the PDB are likely to be wrong (Correspondence, p. 303).

Researchers must bear the responsibility for ensuring that their structures are correct and alerting the community to any issues in a timely manner. An addendum published in this issue (p. 361) provides one example in which electron density should have been more judiciously interpreted, and serves as a useful reminder of the importance of secondary support for structural conclusions. Even when a crystal structure is appropriately interpreted but later superseded by a re-refined or otherwise updated structure, authors should inform their original readers of changes made.

Researchers have many allies in their quest for accuracy. As Brändén and Jones said, "Journals must insist on the publication of enough data for crystallographers to convince the reader that they have a correct structure." We take this role seriously. Since Nature Chemical Biology was launched, it has been our policy that crystal structures should be available in the PDB upon publication (http://www.nature.com/authors/policies/ availability.html). In addition, we have always worked with authors and referees to make sure structural data can be reviewed properly. Referees are asked to look closely at omit maps and structural tables, and they frequently ask to see original data or additional imagery to confirm that the structural conclusions are sound. Since 2013, our sister journal Nature Structural \& Molecular Biology has required authors to include with submission a report from the PDB validation server (http://validate.rcsb. org/), a file that can be generated separately from the deposition process (Nat. Struct. Mol. Biol. 20, 533, 2013). We encourage authors to obtain these reports for their own review, and we welcome your feedback as to whether they should be required at Nature Chemical Biology as well.

The community also has a role to play. As Brändén and Jones advised, “...the readership should be sophisticated enough to judge the quality of the data." Chemical biologists draw extensively on crystal structures to study enzyme active sites, protein-protein interactions, protein- or RNA-ligand complexes and conformational changes. However, if a structure is flawed, research time can be lost chasing down incorrect hypotheses. Thus users must educate themselves on at least the basics of crystallography, such as what conclusions can be drawn depending on structural resolution and how different statistics reflect structural quality. As they begin a project, scientists should look at coordinates with their corresponding density maps, as well as compare multiple structures of the same protein or ligand, if available, to identify variations between structures that could be due to crystal packing or errors in refinement or interpretation. Similarly, if a particular structure will heavily influence a research project, scientists may want to ask a trusted colleague to look at the structure, and follow up on any concerns with the author and/or journal. Chemical biologists and crystallographers should also work together to create or improve validation tools for diverse classes of small molecules and modifications. Finally, educators can incorporate crystal structure analysis into introductory science courses as a way to bring theoretical concepts to life while giving students important practical training.

Crystal structures can be incredibly powerful or frustratingly misleading. Even 25 years later, Brändén and Jones' cautionary note that crystallographic "interpretation remains to some extent subjective" still resonates today, and the interpretation of biomolecular structures will only become more complicated with the continued rise of cryo-electron microscopy, atomic force microscopy and hybrid methods. In the first issue of Nature Structural Biology, the editors suggested that "... the static image of the molecule is rarely an end in itself, but rather a beginning of comprehension" (Nat. Struct. Biol. 1, 1-2, 1994). Here's hoping that the questions your structure raises are scientific and not technical ones. 